\title{
BMJ Open EPISYNC study: predictors of patient- ventilator asynchrony in a prospective cohort of patients under invasive mechanical ventilation - study protocol
}

\author{
Mayson Laercio de Araujo Sousa, ${ }^{\oplus 1,2}$ Rudys Magrans, ${ }^{3,4}$ Fátima K Hayashi, ${ }^{1,2}$ \\ Lluis Blanch, ${ }^{3,4}$ R M Kacmarek, ${ }^{5,6}$ Juliana C Ferreira ${ }^{1}$
}

To cite: Sousa MLA, Magrans R, Hayashi FK, et al. EPISYNC study: predictors of patientventilator asynchrony in a prospective cohort of patients under invasive mechanical ventilation study protocol. BMJ Open 2019;9:e028601. doi:10.1136/ bmjopen-2018-028601

- Prepublication history for this paper is available online. To view these files, please visit the journal online (http://dx.doi. org/10.1136/bmjopen-2018028601).

Received 29 December 2018 Revised 12 March 2019 Accepted 28 March 2019

\section{Check for updates}

(c) Author(s) (or their employer(s)) 2019. Re-use permitted under CC BY-NC. No commercial re-use. See rights and permissions. Published by BMJ.

For numbered affiliations see end of article.

\section{Correspondence to} Mayson Laercio de Araujo Sousa;

mayson.laercio@hc.fm.usp.br

\section{ABSTRACT}

Introduction Patient-ventilator asynchrony is common during the entire period of invasive mechanical ventilation (MV) and is associated with worse clinical outcomes. However, risk factors associated with asynchrony are not completely understood. The main objectives of this study are to estimate the incidence of asynchrony during invasive MV and its association with respiratory mechanics and other baseline patient characteristics.

Methods and analysis We designed a prospective cohort study of patients admitted to the intensive care unit (ICU) of a university hospital. Inclusion criteria are adult patients under invasive MV initiated for less than 72 hours, and with expectation of remaining under MV for more than 24 hours. Exclusion criteria are high flow bronchopleural fistula, inability to measure respiratory mechanics and previous tracheostomy. Baseline assessment includes clinical characteristics of patients at ICU admission, including severity of illness, reason for initiation of MV, and measurement of static mechanics of the respiratory system. We will capture ventilator waveforms during the entire MV period that will be analysed with dedicated software (Better Care, Barcelona, Spain), which automatically identifies several types of asynchrony and calculates the asynchrony index (Al). We will use a linear regression model to identify risk factors associated with Al. To assess the relationship between survival and Al we will use Kaplan-Meier curves, log rank tests and Cox regression. The calculated sample size is 103 patients. The statistical analysis will be performed by the software $\mathrm{R}$ Programming (www.R-project.org) and will be considered statistically significant if the $p$ value is less than 0.05 . Ethics and dissemination The study was approved by the Ethics Committee of Instituto do Coração, School of Medicine, University of São Paulo, Brazil, and informed consent was waived due to the observational nature of the study. We aim to disseminate the study findings through peer-reviewed publications and national and international conference presentations.

Trial registration number NCT02687802; Pre-results.

\section{INTRODUCTION}

Patient-ventilator asynchrony can be defined as a mismatch between the output of a patient's respiratory centre and the
Strengths and limitations of this study

- This cohort study will provide important information about the association between respiratory mechanics and baseline patient characteristics and the incidence of patient-ventilator asynchrony analysed during the entire period of mechanical ventilation.

- Multiple strategies will be put in place to enhance the precision and accuracy of our main predictors and to mitigate the risk of bias.

- The primary outcome will be automatically measured by a validated software, capable of continuously detecting the main types of asynchronies.

- Limitations include the study location in a single centre, and the inability of algorithm of asynchrony to detect flow asynchronies.

programmed delivery of gas from the ventilator. ${ }^{2}$ Asynchrony is a frequent event, present throughout the entire period of mechanical ventilation, although with highly variable frequency, and is associated with worse clinical outcomes, such as prolonged mechanical ventilation, longer intensive care unit (ICU) and hospital stay, respiratory muscle injury, need for tracheostomy and mortality. ${ }^{3-7}$

Factors that influence the incidence of asynchrony can be related to the patient, including severity of illness, patient response to medical treatments and respiratory mechanics. ${ }^{8}$ Investigators assessing patient-ventilator interaction compared four groups of patients with different underling diseases, and found that the incidence of asynchrony was higher in patients with chronic obstructive pulmonary disease (COPD), who already have elevated airway resistance, especially in those with abnormal respiratory system static compliance. ${ }^{9}$ Other studies have also shown increased incidence of asynchrony in COPD patients, associated with respiratory 
system mechanic abnormalities and higher ventilatory demand. ${ }^{3} 10-12$ These findings suggest that the underlying disease and respiratory mechanics may play a role in the incidence of asynchrony. Respiratory mechanics are easy to measure at initiation of mechanical ventilation and throughout the course of mechanical ventilation, allowing the determination of the impact of respiratory mechanics on the development of asynchrony.

The most frequently used method to detect asynchrony is evaluation of ventilator waveforms. However, correct identification requires well trained critical care professionals at the bedside at the time the asynchrony occurs, and is related with a number of factors, including the timing and duration of waveform evaluations. ${ }^{13-15}$ Hence, monitoring technologies have been recently developed to continuously and automatically detect patient-ventilator asynchrony, especially ineffective effort and double triggering. One of those, recently validated by Blanch and colleagues (Better Care, Barcelona, Spain) can automatically detect the majority of asynchronies with accuracy similar to that of expert intensivists, with good sensitivity $(91.5 \%)$ and specificity $(96.2 \%) .{ }^{1617}$

Therefore, we designed an observational cohort study to estimate the incidence of patient-ventilator asynchrony during invasive mechanical ventilation and its association with respiratory mechanics at intubation and others baseline patient characteristics. We hypothesise that abnormal baseline respiratory mechanics-increased airway resistance and intrinsic positive end-expiratory pressure (PEEP) and decreased static compliance-are associated with an increase in the patient-ventilator asynchrony index (AI). The secondary objectives include: (1) measurement of the level of agreement between clinical examination and visual inspection of the ventilator screen at the bedside, with the AI automatically calculated by continuous analysis of ventilator waveforms and evaluation of the performance of clinical examination in the assessment of asynchrony at the bedside; and (2) assessment of the relationship between the AI with ventilator-free days, and ICU and hospital survival. This paper describes the study design and the EPIdemiology of a SYNChrony (EPISYNC) protocol.

\section{METHODS}

\section{Study location and design}

We designed a prospective cohort study, including patients under recent invasive mechanical ventilation, aged $\geq 18$ years, and with expectation of mechanical ventilation for more than 24 hours after enrolment.

Patients will be recruited in the Respiratory ICU of Hospital das Clínicas, School of Medicine, University of São Paulo, Brazil.

\section{Eligibility criteria}

The inclusion and exclusion criteria are enumerated in box 1. Exclusion criteria were designed to ensure precision and accuracy of the principal predictive variables

\section{Box 1 Participant inclusion and exclusion criteria}

Inclusion criterialnclusion criteria

- Mechanical ventilation initiated within 72 hours.

- Expectation of mechanical ventilation for more than 24 hours after enrolment.

- Age $\geq 18$ years.

Exclusion criteriaExclusion criteria

- High flow bronchopleural fistula (pneumothorax with large air leaks from the chest tube and continuous air bubbling).

- Thoracic or abdominal deformities that could compromise the accuracy of respiratory mechanics measurement.

- Inability to measure respiratory mechanics (eg, death before respiratory mechanics measurement or physician's decision not to use sedation and/or neuromuscular blockage).

Tracheostomy.

(respiratory mechanics) and prevent possible missing data. Tracheostomy will be one of study outcomes, so patients tracheostomised before ICU admission will be excluded.

\section{Sampling and screening process}

The target sample size for EPISYNC is 103 participants (see below, statistical analysis), to be recruited in the Respiratory ICU of School of Medicine, University of São Paulo, Brazil, and we will use consecutive sampling. Daily, one of the investigators will screen all patients admitted in the ICU; patients fulfilling all inclusion criteria and no exclusion criteria will be included in study. For patients submitted to more than one episode of mechanical ventilation during their hospital stay, only the first episode will be registered.

\section{Study measures and data collection}

We will describe the data collection in three steps, as shown in figure 1: baseline, follow-up during mechanical ventilation and postextubation follow-up. Main study measures and data collection schedule are shown in table 1 .

\section{Baseline data}

Baseline respiratory mechanics-static compliance, airway resistance and intrinsic PEEP-will be calculated within 72 hours postintubation, as soon as the patient is included in the study. We will measure static properties of the respiratory system consistent with the ICU's routine protocol for all invasively ventilated patients at admission. Measurements will be performed 15 min after endotracheal suctioning, using volume-controlled ventilation, with tidal volume $\left(\mathrm{V}_{\mathrm{T}}\right)$ of $6 \mathrm{~mL} / \mathrm{kg}$ of predicted body weight, square flow waveform and inspiratory flow of 0.5 $\mathrm{l} / \mathrm{s}$, following the protocol used in our ICU. These standardised ventilator settings will be used only to measure respiratory mechanics. After the measurements, the mode of ventilation and its adjustments will be left to the ICU team's discretion. We will calculate predicted body weight using the following equations (1): 


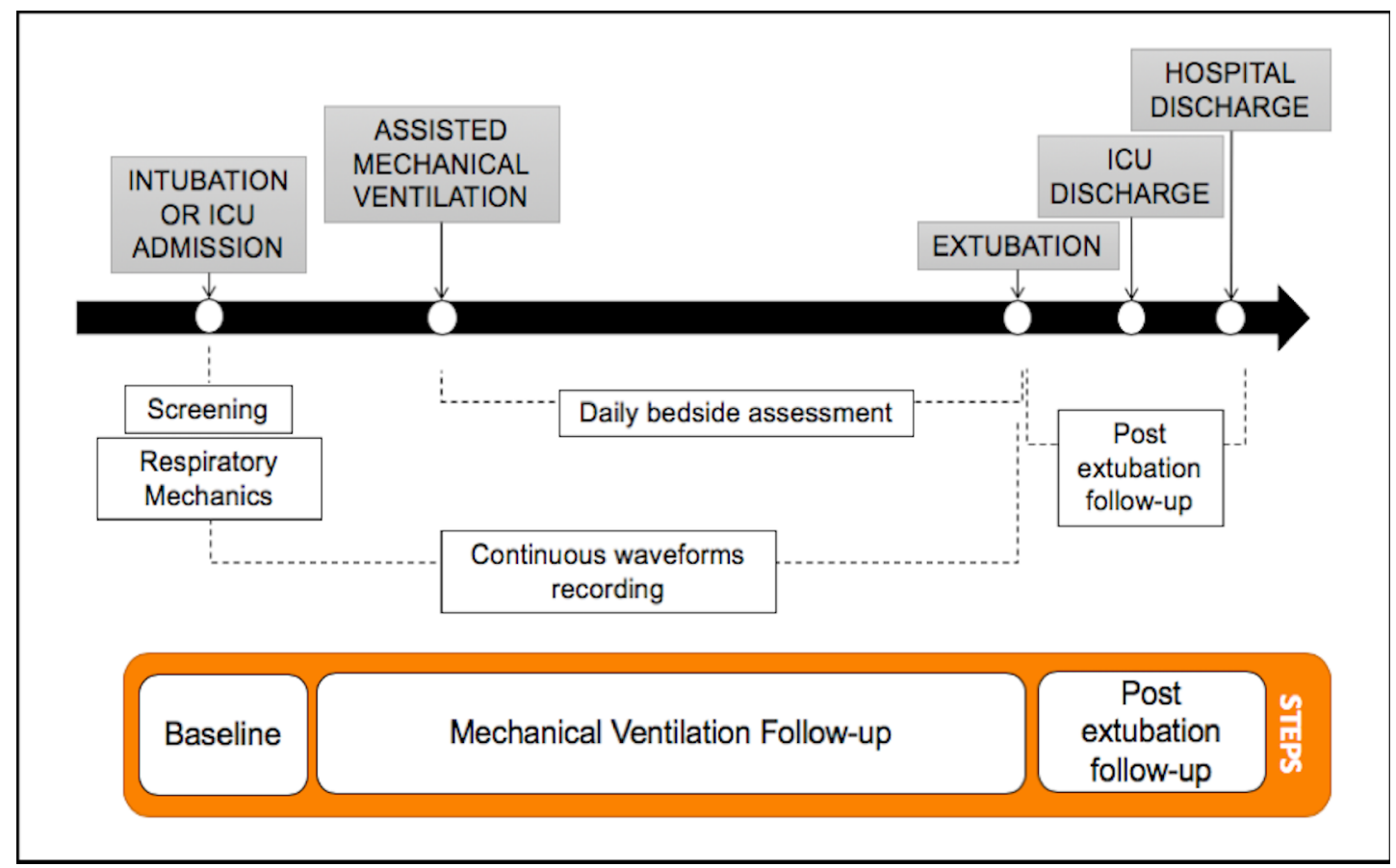

Figure 1 Overview of EPISYNC protocol. ICU, intensive care unit.

$50+2.5\{[\operatorname{height}(\mathrm{cm}) \times 0.394]-60\}$, for male

$45.5+2.5\{[$ height $(\mathrm{cm}) \times 0.394]-60\}$, for female

We will perform an end-inspiratory pause of $4 \mathrm{~s}$ and measure peak pressure $\left(\mathrm{P}_{\text {peak }}\right)$ and plateau pressure $\left(\mathrm{P}_{\text {plat }}\right)$; then we will perform an end-expiratory pause of $4 \mathrm{~s}$ to measure total positive end-expiratory pressure $\left(\mathrm{PEEP}_{\text {total }}\right)$, defined as the airway pressure at the end of the expiratory pause. All variables will be measured three times and the mean values will be used. The equations used to calculate static compliance $\left(\mathrm{C}_{\text {stat }}\right)(2)$, airway resistance $\left(\mathrm{R}_{\mathrm{aw}}\right)$ (3) and intrinsic PEEP (PEEP $)_{i}$ (4) are:

$$
\begin{gathered}
\text { Cstat }=\frac{V T}{\text { Pplat }- \text { PEEPtotal }} \\
\text { Raw }=\frac{\text { Ppeak }- \text { Pplat }}{\text { Flow }} \\
\text { PEEP } i=\text { PEEPtotal }- \text { PEEPsetting }
\end{gathered}
$$

If the patient continues receiving neuromuscular blocking agents on the following days, the respiratory mechanics will be recalculated daily.

\section{Follow-up during mechanical ventilation}

The primary outcome measure is the AI, defined as the number of asynchronous events divided by the total number of ventilator cycles and wasted efforts multiplied by $100 .^{3}$ To estimate AI we will use the validated software Better Care (Barcelona, Spain). ${ }^{16}$ The software will continuously record airflow, airway pressure and tidal volume from study inclusion until liberation from the ventilator, tracheostomy, death or 28 days of mechanical ventilation. Secondary outcomes including ventilator-free days, tracheostomy and survival will be recoded on all patients. The ventilators available in our ICU are Servo-i (Maquet) and Puritan Bennet 840 (Covidien).
Trained respiratory therapists will use a checklist to daily access the presence of clinically relevant patient-ventilator asynchrony on days when the patient is not receiving neuromuscular blocking agents. The evaluation will be done during the morning, with the patient in the semirecumbent position, 15 min after airway suctioning. The check list includes observation of clinical signs of respiratory distress (box 2) and waveform observation on the ventilator screen. The respiratory therapists will be trained to learn to recognise missed efforts, flow asynchrony, autotriggering, double triggering, short and prolonged cycling. They will observe the waveforms for $5 \mathrm{~min}$, and then make a clinical judgement if asynchronous events appear to be present in more than $10 \%$ of the cycles.

The daily checklist will also include Sequential Organ Failure Assessment, Richmond Agitation and Sedation Scale, Behavioural Pain Scale and the Confusion Assessment Method for the ICU.

\section{Ventilator parameters}

As mentioned above, we will use a software (Better Care, Barcelona, Spain) capable of acquiring, analysing and recording signals from digital medical devices. Mechanical ventilators and bedside monitors will be connected to the acquisition software using a remote access server (ED41000P2-01-Lantronix, Irvine, California, USA).

To calculate AI, the software continuously captures the digital output of the mechanical ventilator and associates each acquired waveform with the parameter it represents (airflow, air pressure and tidal volume). To identify asynchronous events, the first step is to identify the mechanical ventilation mode (continuous positive 


\begin{tabular}{|c|c|c|c|}
\hline & Baseline & $\begin{array}{l}\text { Mechanical } \\
\text { ventilation } \\
\text { follow-up }\end{array}$ & $\begin{array}{l}\text { Postextubation } \\
\text { follow-up }\end{array}$ \\
\hline Demographics & $x$ & & \\
\hline Height & $x$ & & \\
\hline Weight & $x$ & & \\
\hline Cause of ICU admission & $x$ & & \\
\hline Comorbidities & $x$ & & \\
\hline Smoking history & $x$ & & \\
\hline SAPS 3 & $x$ & & \\
\hline Cause of intubation & $\mathrm{x}$ & & \\
\hline ARDS criteria at admission & $x$ & & \\
\hline Static compliance & $x$ & & \\
\hline Airway resistance & $x$ & & \\
\hline Intrinsic PEEP & $x$ & & \\
\hline $\mathrm{PaO}_{2} / \mathrm{FiO}_{2}$ & $x$ & $x$ & \\
\hline Sedation scale (RASS) & $x$ & $x$ & \\
\hline Glasgow Coma Scale & $x$ & $x$ & \\
\hline Delirium & & $x$ & \\
\hline $\begin{array}{l}\text { Behavioural Pain Scale } \\
\text { (BPS) }\end{array}$ & & $x$ & \\
\hline
\end{tabular}

\begin{tabular}{lll}
\hline Pneumothorax & $\mathrm{X}$ & \\
\hline SOFA score & $\mathrm{X}$ & \\
\hline Humidification system & $\mathrm{X}$ & \\
$\begin{array}{l}\text { Visual perception of } \\
\text { asynchrony on ventilator } \\
\text { screen }\end{array}$ & $\mathrm{X}$ & \\
\hline $\begin{array}{l}\text { Clinical signs of asynchrony } \\
\text { Ventilator settings }\end{array}$ & $\mathrm{X}$ & \\
\hline $\begin{array}{l}\text { Duration of mechanical } \\
\text { ventilation }\end{array}$ & $\mathrm{X}$ & $\mathrm{X}$ \\
\hline $\begin{array}{l}\text { Use of NIV postextubation } \\
\text { Tracheostomy }\end{array}$ & $\mathrm{X}$ \\
\hline $\begin{array}{l}\text { Mechanical ventilation free } \\
\text { days }\end{array}$ & $\mathrm{X}$ \\
\hline $\begin{array}{l}\text { Length of ICU stay } \\
\text { Length of hospital stay }\end{array}$ & $\mathrm{X}$ \\
\hline $\begin{array}{l}\text { Discharge status (dead or } \\
\text { alive) }\end{array}$ & $\mathrm{X}$ \\
\hline
\end{tabular}

ARDS, acute respiratory distress syndrome; ICU, intensive care unit; NIV, non-invasive ventilation; PEEP, positive end-expiratory pressure; RASS, Richmond Agitation and Sedation Scale; SAPS3, Simplified Acute Physiology Score 3; SOFA, Sequential Organ Failure Assessment.

airway pressure; volume control ventilation (VCV); pressure-controlled ventilation (PCV) and pressure support ventilation (PSV)), according to the pattern of inspiratory time (IT), flow, airway pressure and tidal volume. Then, in each breath, the software investigates the presence of ineffective expiratory effort and double triggering in VCV, PCV and PSV; short cycling and prolonged cycling in PSV, as shown in table 2 and figure 2.

Postextubation follow-up

We will follow patients until hospital discharge or death within the hospital, and will compute the duration of
Box 2 Clinical signs of respiratory distress/asynchrony

- Tachypnoea.

- Use of accessories muscles.

- Diaphoresis.

- Paradoxical diaphragmatic movement.

- Subdiaphragmatic retraction.

- Suprasternal, supraclavicular or intercostal retraction.

- Cyanosis.

- Tachycardia.

- Oxygen desaturation.

Others.

Adapted from Pierson. ${ }^{23}$

mechanical ventilation, ventilator-free days, defined as the number of days alive and off the ventilator in the first 28 days of ICU stay, the use of postextubation non-invasive ventilation, reintubation, tracheostomy, ICU and hospital length of stay and mortality.

\section{Statistical analysis}

Analytic plan

Categorical variables will be presented as proportions and continuous variables will be reported as mean and SD or medians and IQR.

To evaluate relationship between static compliance, airway resistance and intrinsic PEEP and the AI, all continuous variables, we will use linear regression. To evaluate the relationship between $\mathrm{AI}$ and other categorical variables, we will dichotomise $\mathrm{AI}$ into $\mathrm{AI} \leq 10 \%$ and $\mathrm{AI}>10 \%,{ }^{4}$ and use $\chi^{2}$ test and logistic regression.

We will calculate AI for the entire duration of mechanical ventilation, the daily AI, and hourly AI, summing all types of asynchrony, and by each type of asynchrony. We

Table 2 Ventilatory data analysis and processing by Better Care

Ineffective Abrupt decreases or increases in expiratory inspiratory flow by searching for a positive maximum effort value followed by a negative minimum value on the first arm of the flow curve and then evaluating the deviations of these values against a monoexponential curve representing the theoretical mean expiratory flow. (figure $2 \mathrm{~A}$ )

Double Two consecutively effective cycles that are triggering separated by an expiratory time less than half the mean $\mathrm{Ti}$ (over the last $20 \mathrm{Ti}$ ), the first cycle triggered by the patient. (figure $2 \mathrm{~B}$ )

Short cycling In PSV, Ti of the current breath is $50 \%$ shorter than the averaged $\mathrm{Ti}$ (over the last 20 Ti). (figure 2C)

$\begin{array}{ll}\text { Prolonged } & \text { In PSV, Ti of the current breath is 200\% } \\ \text { cycling } & \text { larger than the averaged Ti (over the last 20 } \\ & \text { Ti). (figure 2D) }\end{array}$

PCV, pressure-controlled ventilation.; PSV, pressure support ventilation; Ti, inspiratory time; VCV, volume control ventilation, 


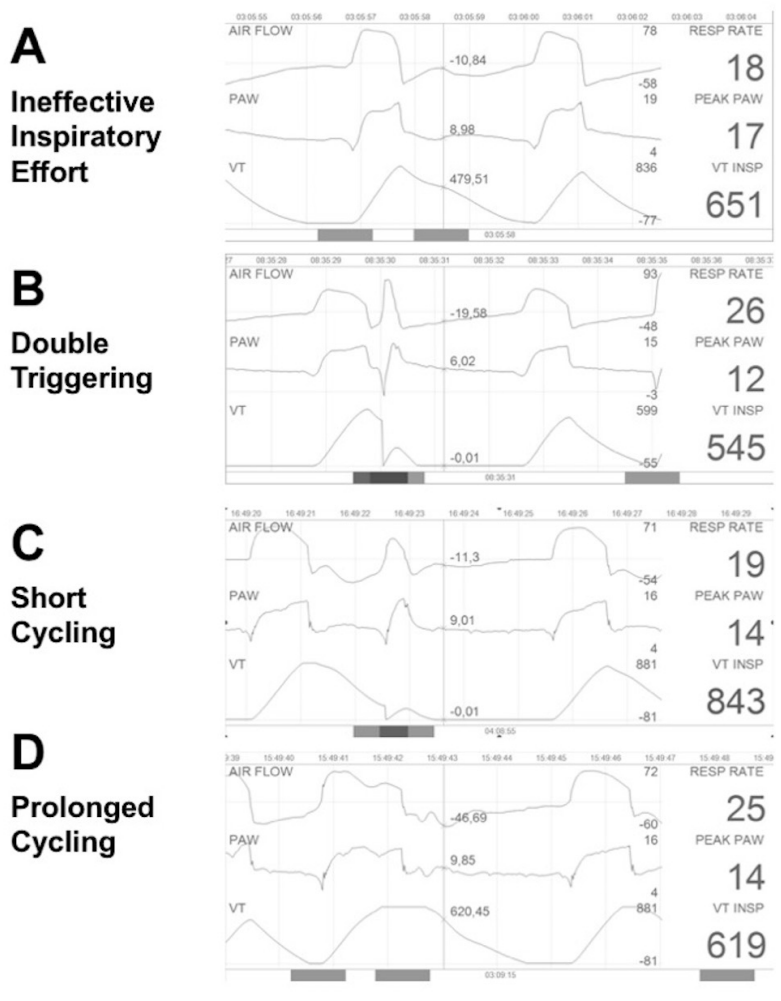

Figure 2 Examples of asynchrony events detected by Better Care. INSP, inspiratory; PAW, airway pressure; RESP, respiratory; VT, tidal volume.

will also explore the role of clusters of ineffective effort and double triggering on clinical outcomes. ${ }^{18} 19$

To assess the relationship between survival and AI we will use Kaplan-Meier curves, log rank tests and Cox regression.

The statistical analysis will be performed by the software R Programming (R Core Team, Vienna, Austria; URL: https://www.R-project.org) and will be considered statistically significant if the $p$ value is less than 0.05 .

\section{Sample size}

We used G*Power 3.1 to calculate sample size. We estimated an effect size of 0.11 , based on an expected increase in $\mathrm{R}^{2}$ up to 0.10 . Using an $\alpha$ of 0.05 , a power of 0.8 and testing three main predictors (static compliance, airway resistance and intrinsic PEEP) and up to five more baseline predictors, we calculated a total sample size of 103 patients.

\section{Quality control}

\section{Data collection and management}

To enhance the precision and accuracy of our main predictors, we will measure baseline respiratory mechanics three times and use the mean values. Given that this is an observational study, the choice of sedatives and neuromuscular blockage agents and doses used will be at ICU physician's discretion, but we will use train-of-four monitoring (SunStim Plus, SunMed, Michigan, USA) to determine the depth of neuromuscular blockage. Furthermore, we developed a manual of operations for all study-related activities.
We will collect and manage all study data using Research Electronic Data Capture (REDCap) electronic data capture tools hosted at Hospital das Clínicas da Faculdade de Medicina da Universidade de Sao Paulo, Brazil. REDCap is a secure, web-based application designed to support data capture for research studies, providing: (1) an intuitive interface for validated data entry; (2) audit trails for tracking data manipulation and export procedures; (3) automated export procedures for seamless data downloads to common statistical packagesand (4) procedures for importing data from external sources. ${ }^{20}$

The final database will be cleaned, verified and archived. One official copy of all the study data and master data dictionary will be maintained and updated regularly by study data analysts. One copy of the backups will be saved in an external drive. The files can be shared only by authorised study personnel.

\section{Patient and public involvement}

Patients were not involved in the development of the research question or the design of this study.

\section{Ethics and dissemination}

Because we routinely perform respiratory mechanics measurements in our ICU for all patients at admission using sedation and neuromuscular blockage if necessary, and the study was non-interventional and did not interfere with usual care, informed consent was waived. The protocol is registered in the international platform ClinicalTrials.gov.

We will report study results in accordance to recommended guidelines for reporting observational studies, the Strengthening The Reporting of Observational Studies in Epidemiology. ${ }^{21}$

The results of the primary study will be published in a peer-reviewed journal. Separate manuscripts will be written on secondary objectives and exploratory analysis and will also be submitted for publication in peer-reviewed journals.

\section{Current status}

Recruitment of participants started at September 2016, and we expect to complete this study by June 2019 .

\section{DISCUSSION}

The EPISYNC study will provide important insights into the association between baseline patient characteristics, including respiratory mechanics, and the incidence of patient-ventilator asynchrony analysed during the entire period of mechanical ventilation. Also, we aim to provide further insight into the association between asynchrony and clinical outcomes, such as ventilator-free days, and ICU and hospital survival. These results will contribute to the knowledge base regarding asynchrony and its epidemiology, and may influence ventilator guidelines to improve synchrony in critical ill patients. 
Our study has some limitations. First, the study is confined to a single centre, so the incidence of asynchrony will be influenced by the centre's practice, specific ventilators used and the manner in which ventilator management is provided. In addition, respiratory mechanics measurement using neuromuscular blocking agents is not a universal standard and will not always be possible. Second, the algorithm of asynchrony detection has some limitations detecting short cycling and prolonged cycling, and reverse triggering will be detected only when it results in double triggering. Short cycling and prolonged cycling will not be detected in extreme cases of alternating breaths of very short $\mathrm{Ti}$ with breaths with prolonged Ti. Furthermore, the Better Care software is not able to detect flow asynchronies and auto triggering. However, several studies have shown that these types of asynchrony have a very low incidence and that ineffective effort and double triggering are the most important types of asynchrony to calculate AI, and for these types of asynchrony our methodology is very accurate. And finally, the sample size was calculated based on the primary outcome variable and predictors, so we may be underpowered to detect differences in mortality.

Nonetheless, we believe that our results will be generalisable to ICUs in low and middle incoming countries, where the burden of critically ill diseases is high and mortality rates of patients requiring ventilatory support is elevated. ${ }^{22}$

\section{Author affiliations}

${ }^{1}$ Divisao de Pneumologia, Instituto do Coracao, Hospital das Clinicas HCFMUSP, Faculdade de Medicina Universidade de Sao Paulo, Sao Paulo, Brazil ${ }^{2}$ Serviço de Fisioterapia, Instituto do Coracao, Hospital das Clinicas HCFMUSP, Faculdade de Medicina Universidade de Sao Paulo, Sao Paulo, Sao Paulo, Brazil ${ }^{3}$ Critical Care Center, Hospital Universitari Parc Taulí, Institut d'Investigació i Innovació Parc Taulí I3PT, Universitat Autònoma de Barcelona, Sabadell, Spain ${ }^{4}$ Centro de Investigación Biomédica en Red de Enfermedades Respiratorias (CIBERES), Instituto de Salud Carlos III, Sabadell, Spain

${ }^{5}$ Department of Respiratory Care, Massachusetts General Hospital, Boston, Massachusetts, USA

${ }^{6}$ Department of Anesthesiology, Massachusetts General Hospital, Boston, Massachusetts, USA

Contributors MLdAS and JCF conceived and designed the protocol, drafted the manuscript and were involved in acquisition of funding. MLdAS organised and performed the initial analysis of the data. All authors participated in the final analysis of the data. RMK, LB, RM and FKH participated in the critical revision of the manuscript for methodological and intellectual content. All authors approved the final version of the manuscript.

Funding The Episync study is supported by Fundação de Amparo à Pesquisa do Estado de São Paulo (FAPESP), grant number 2015/19122-4.

Competing interests RMK reports grants and personal fees from Medtronic, personal fees from Orange Medical, grants from Venner Medical, outside the submitted work. LB is inventor of one Corporació Sanitaria Parc Taulí owned US patent: 'Method and system for managed related patient parameters provided by a monitoring device,' US Patent No. 12/538,940, founder of BetterCare S.L. which is a research and development company, start up of Corporació Sanitària Parc Taulí. JCF reports grants from FAPESP (Brazilian funding agency), during the conduct of the study; personal fees from Medtronic, outside the submitted work.

Patient consent for publication Not required.

Ethics approval The EPISYNC protocol was approved by the institutional review board of Instituto do Coração, School of Medicine, University of São Paulo, Brazil (reference number 1.327.840).
Provenance and peer review Not commissioned; externally peer reviewed.

Open access This is an open access article distributed in accordance with the Creative Commons Attribution Non Commercial (CC BY-NC 4.0) license, which permits others to distribute, remix, adapt, build upon this work non-commercially, and license their derivative works on different terms, provided the original work is properly cited, appropriate credit is given, any changes made indicated, and the use is non-commercial. See: http://creativecommons.org/licenses/by-nc/4.0/.

\section{REFERENCES}

1. Sassoon CS, Foster GT. Patient-ventilator asynchrony. Curr Opin Crit Care 2001;7:28-33.

2. Thille AW, Brochard L. Promoting Patient-Ventilator Synchrony. Clin Pulm Med 2007;14:350-9.

3. Chao DC, Scheinhorn DJ, Stearn-Hassenpflug M. Patient-ventilator trigger asynchrony in prolonged mechanical ventilation. Chest 1997;112:1592-9.

4. Thille AW, Rodriguez P, Cabello B, et al. Patient-ventilator asynchrony during assisted mechanical ventilation. Intensive Care Med 2006;32:1515-22.

5. de Wit M, Miller KB, Green DA, et al. Ineffective triggering predicts increased duration of mechanical ventilation. Crit Care Med 2009;37:2740-5.

6. Blanch L, Villagra A, Sales B, et al. Asynchronies during mechanical ventilation are associated with mortality. Intensive Care Med 2015;41:633-41.

7. Ferreira JC, Diniz-Silva F, Moriya HT, et al. Neurally Adjusted Ventilatory Assist (NAVA) or Pressure Support Ventilation (PSV) during spontaneous breathing trials in critically ill patients: a crossover trial. BMC Pulm Med 2017;17:139.

8. Branson RD, Blakeman TC, Robinson BR. Asynchrony and dyspnea. Respir Care 2013;58:973-89.

9. Nava S, Bruschi C, Fracchia C, et al. Patient-ventilator interaction and inspiratory effort during pressure support ventilation in patients with different pathologies. Eur Respir J 1997;10:177-83.

10. Leung P, Jubran A, Tobin MJ. Comparison of assisted ventilator modes on triggering, patient effort, and dyspnea. Am J Respir Crit Care Med 1997;155:1940-8.

11. Parthasarathy S, Jubran A, Tobin MJ. Cycling of inspiratory and expiratory muscle groups with the ventilator in airflow limitation. $A m$ $J$ Respir Crit Care Med 1998;158:1471-8.

12. Jolliet $P$, Tassaux $D$. Clinical review: patient-ventilator interaction in chronic obstructive pulmonary disease. Crit Care 2006;10:236.

13. Colombo D, Cammarota G, Alemani M, et al. Efficacy of ventilator waveforms observation in detecting patient-ventilator asynchrony. Crit Care Med 2011;39:2452-7.

14. Chacón E, Estruga A, Murias G, et al. Nurses' detection of ineffective inspiratory efforts during mechanical ventilation. Am J Crit Care 2012;21:e89-93.

15. Ramirez II, Arellano DH, Adasme RS, et al. Ability of ICU HealthCare Professionals to identify patient-ventilator asynchrony using waveform analysis. Respir Care 2017;62:144-9.

16. Blanch L, Sales B, Montanya J, et al. Validation of the Better Care ${ }^{\circledR}$ system to detect ineffective efforts during expiration in mechanically ventilated patients: a pilot study. Intensive Care Med 2012;38:772-80.

17. Murias G, Montanyà J, Chacón E, et al. Automatic detection of ventilatory modes during invasive mechanical ventilation. Crit Care 2016;20:1-7.

18. Vaporidi K, Babalis D, Chytas A, et al. Clusters of ineffective efforts during mechanical ventilation: impact on outcome. Intensive Care Med 2017;43:184-91.

19. de Haro C, López-Aguilar J, Magrans R, et al. Double cycling during mechanical ventilation. Crit Care Med 2018;46:1385-92.

20. Harris PA, Taylor R, Thielke R, et al. Research electronic data capture (REDCap)--a metadata-driven methodology and workflow process for providing translational research informatics support. J Biomed Inform 2009;42:377-81.

21. von Elm E, Altman DG, Egger M, et al. The Strengthening the Reporting of Observational Studies in Epidemiology (STROBE) statement: guidelines for reporting observational studies. J Clin Epidemiol 2008;61:344-9.

22. Azevedo LC, Park M, Salluh JI, et al. Clinical outcomes of patients requiring ventilatory support in Brazilian intensive care units: a multicenter, prospective, cohort study. Crit Care 2013;17:R63.

23. Pierson DJ. Patient-ventilator interaction. Respir Care 2011;56:214-28. 